\title{
FETOMETRIA ULTRASSONOGRÁFICA EM PORCAS GESTANTES COMO MÉTODO COMPLEMENTAR PARA AVALIAÇÃO DA MORFOLOGIA E DETERMINAÇÃO DA IDADE FETAL
}

\author{
DANEZE, Edmilson Rodrigo ${ }^{1}$ \\ LÉGA , Elzylene ${ }^{2}$ \\ PINTO, Mildre Loraine ${ }^{3}$
}

Recebido em: $2012-04-17$

Aprovado em: $2012-10-23$

ISSUE DOI: $10.3738 / 1982.2278 .744$

\begin{abstract}
RESUMO: Frente ao avanço na utilização das biotécnicas reprodutivas e aos poucos dados referentes ao desenvolvimento gestacional em suínos, faz-se necessária a aplicação de método eficiente de diagnóstico de gestação e da estimativa de idade fetal. Assim, foram submetidas a exames ultrassonográficos, em modo-B, sete fêmeas suínas gestantes, da raça Landrace, em diferentes estádios gestacionais com objetivo de diagnosticar a gestação e estudar parâmetros sobre o desenvolvimento embrionário e/ou fetal dos leitões. Concluiu-se que a ultrassonografia mostrou ser um método bastante acurado e apropriado no diagnóstico gestacional e na avaliação da biometria e morfologia fetais, sendo que o diâmetro biparietal teve o maior número de mensurações quando comparado às demais variáveis, sendo as mesmas consideradas acuradas em virtude das datas de cobertura e de parto serem conhecidas. No entanto, novos estudos devem ser realizados na espécie suína, comparando-se a técnica de biometria fetal em diferentes raças e em diferentes estádios gestacionais.
\end{abstract}

Palavras-chave: Suíno. Gestação. Biometria fetal. Obstetrícia. Ultrassonografia.

\section{ULTRASONOGRAPHY FETOMETRY IN PREGNANT SOWS AS A COMPLEMENTARY METHOD TO EVALUATE THE MORPHOLOGY AND DETERMINATION OF FETAL AGE}

SUMMARY: Faced with the advance in the use of reproductive biotechniques and few data reporting of gestational development in swines, it is necessary to apply efficient method of pregnancy diagnosis and estimation of fetal age. Thus, were subjected to ultrasound, B-mode, seven pregnant sows, Landrace, at different stages of pregnancy in order to diagnose pregnancy and study parameters on the development of embryonic and fetal pigs. It was concluded that ultrasonography has proved to be a very suitable and accurate method for diagnosing pregnancy and in the assessment of biometry fetal and morphology, and the biparietal diameter had the largest number of measurements when compared with the other variables. Such measures were considered accurate because the dates of mating and birthing are known. However, further studies should be performed in swine, compared to the technique of fetal biometry in different races and different stages of pregnancy.

Keywords: Swine. Gestation. Fetal biometrics. Obstetrics. Ultrasonography.

\section{INTRODUÇÃO}

A gestação da fêmea suína dura, em média, 115 dias, com variações para mais ou menos três dias, sendo fundamentais os cuidados durante esse período para potencializar a produtividade, já que as mesmas passam dois terços de sua vida útil em gestação e o desempenho reprodutivo tem grande

\footnotetext{
${ }^{1}$ Aprimorando em Clínica e Cirurgia Veterinária- Faculdade Dr. Francisco Maeda-FE

2 Professora Doutora. Setor de Diagnóstico por Imagem. Departamento de Clínica e Cirurgia Veterinária. Coordenadora do curso de Medicina Veterinária Faculdade Dr. Francisco Maeda-FE

${ }^{3}$ Médica Veterinária
} 
importância na eficiência econômica da atividade (SILVEIRA et al., 1998; ABUD et al., 2009).

Frente ao avanço na utilização de programas de indução e/ou sincronização de cios, desenvolvimento de métodos de inseminação artificial (IA) e transferência de embriões, faz-se necessário o uso de método eficiente de diagnóstico de gestação, bem como de avaliação da morfologia e estimativa da idade fetal (GRANADOS et al., 2006; ABUD et al., 2009).

A determinação do diagnóstico gestacional positivo ou negativo representa um considerável fator econômico e uma importante ferramenta para o manejo reprodutivo (JAINUDEEN; HAFEZ, 2004; ABREU, 2006) devendo ser realizado o mais precoce possível e, assim, realizar nova IA nas fêmeas não gestantes ou descartá-las quando houver recidivas de diagnósticos negativos (SILVEIRA et al., 1998; ABUD et al., 2009). O não retorno ao estro de 18 a 24 dias após a cobertura ou IA, supõe que a fêmea suína esteja gestante, sendo o teste de pressão lombar um dos métodos mais precoces de diagnóstico gestacional, no entanto, o aumento no diâmetro e presença de frêmito na artéria uterina média, detectado por palpação retal, também podem ser evidência de gestação (SILVEIRA et al. 1998; WENZEL, 2002; JAINUDEEN; HAFEZ, 2004).

O advento da ultrassonografia (US) na medicina veterinária, tornou a identificação de afecções, o diagnóstico da gestação e o acompanhamento do desenvolvimento fetal cada vez mais rápido e eficaz (VIANA et al., 1998; PEREIRA et al., 2001), de forma não invasiva (BALL; PETERS, 2006) e sem prejuízo aos fetos (WOLF; GABALDI, 2002), permitindo ainda detectar o momento da ovulação através da técnica transretal e viabilizando o uso da IA (PEREIRA et al., 2001). Desta forma, o método se tornou pedido comum aos ultrassonografistas veterinários, sendo que a visibilização do útero aumentado e a identificação de uma ou mais vesículas gestacionais confirmam a condição gestacional (NYLAND; MATTOON, 2005; KEALY; McALLISTER, 2005; FELICIANO et al., 2008).

Economicamente acessível, a US é apropriada para detectar porcas gestantes em períodos bem precoces (KAUFFOLD et al., 1997; WILLIAMS et al., 2001; ROMERO, 2006), com visibilização de imagens anatômicas e morfológicas imediatas e de alta qualidade, mesmo em movimento, através de secções transversais, sagitais ou horizontais, por meio de varredura (COSTA, 2002; MACHADO et al, 2002; NYLAND; MATTOON, 2005), sendo eficaz a partir de 30 dias de gestação (WENZEL, 2002). No entanto, foram citadas referências de diagnóstico pelo modo-B com segurança a partir de 22 dias após a cobertura ou IA (JAINUDEEN; HAFEZ, 2004; ABUD et al., 2009).

MORENO et al. (1996) referiram a possibilidade de aferir comprimento de ossos longos, distância interorbital, medidas de crânio, diâmetro do tórax e abdômen e, relacionando esses valores com os dias antes do parto, revelaram que a fetometria é mais apropriada para a determinação da idade gestacional e a previsão do parto do que a mensuração da frequência cardíaca fetal. Uma vez que as estruturas ósseas fetais possam ser visibilizadas torna-se possível calcular a idade gestacional por meio de mensurações fetais, fato importante quando se desconhece a data de acasalamento, permitindo prever a data do parto e manter a fêmea sob manejo adequado (REICHLE, HABEL, 1991; BRETZLAFF et al., 1993).

Nucleus Animalium, v.4, n.2, nov.2012 
A US, por ser método rápido, prático e satisfatório para determinar a fase de desenvolvimento fetal (LÉGA, 2000; TORRES, 2008), torna-se um instrumento importante na detecção de distúrbios obstétricos como desenvolvimento embrionário retardado, malformações fetais e morte fetal, além de aborto e reabsorção embrionária, trazendo vantagens significativas e palpáveis à exploração animal (FERNANDES, 1996). No entanto, muitas dúvidas ainda precisam ser respondidas, tais como influência da raça no tamanho fetal, influência do tamanho da prole no tamanho fetal e influência da raça na curva de desenvolvimento durante o período fetal (NYLAND; MATTOON, 2005).

Na veterinária, caninos e felinos são as espécies que mais usufruem da técnica ultrassonográfica na rotina clínica. Enquanto que em ruminantes e equinos, o método tem otimizado as biotécnicas relacionadas à reprodução. No entanto, poucos são os dados disponíveis sobre o acompanhamento do desenvolvimento fetal em suínos. Assim, o objetivo deste estudo foi realizar fetometria em porcas de diferentes fases gestacionais como estudo complementar para avaliação da morfologia e determinação da idade fetal nesta espécie.

\section{MATERIAL E MÉTODOS}

Foram utilizadas sete fêmeas suínas gestantes, da raça Landrace, pertencentes ao Setor de Suinocultura da Faculdade Dr. Francisco Maeda (FAFRAM/FE), localizada em Ituverava-SP, em diferentes estádios gestacionais, as quais foram submetidas a exame ultrassonográfico para diagnóstico de gestação, avaliação da morfologia fetal e fetometria, sendo que a data da IA foi considerada o dia zero da gestação, valendo salientar que houve preocupação em zelar pelo bem-estar dos animais e, para tanto, nenhuma alteração foi feita no manejo do setor.

A avaliação ultrassonográfica foi realizada em modo-B empregando-se aparelho Pie-Medical Aquila/Falcon, com transdutor eletrônico setorial de 5,0 MHz de frequência, posicionado em região abdominal lateral (Fig. 2), paralela à cadeia mamária, sob lubrificação com gel comercial, mantendo-se o animal em decúbito lateral ou posição quadrupedal, sendo feitas varreduras sequenciais em secções transversal, sagital ou coronal. O abdômen foi examinado em ambos os antímeros, procurando evidenciar o volume e conteúdo uterino, bem como as vesículas embrionárias e o crescimento fetal.

Figura 1. Imagem fotográfica ilustrando o posicionamento do transdutor (seta) durante avaliação ultrassonográfica em porca gestante.

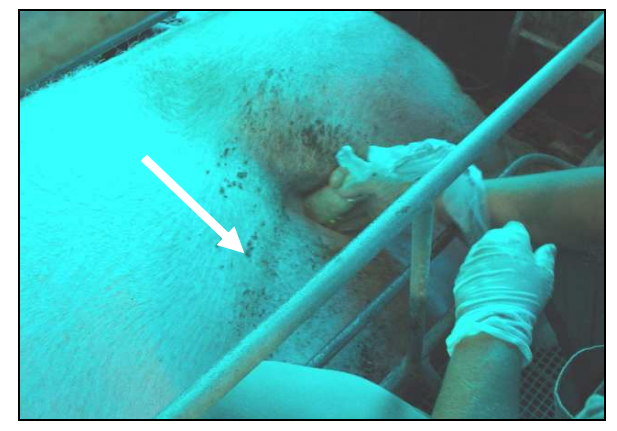

Fonte: Setor de Suinocultura. FAFRAM. Ituverava-SP. 2010.

Nucleus Animalium, v.4, n.2, nov.2012 
Os exames fetométricos seguiram uma sequência padrão de anotações conforme proposto por LÉGA (2000), sendo que, nesse estudo, procurou-se avaliar os seguintes parâmetros: espessura do endométrio (End), diâmetro da vesícula gestacional (DVG), comprimento crânio-caudal (CCC), diâmetro do tórax (DT), diâmetro abdominal transversal (DAT), diâmetro orbital (DO), diâmetro biorbital (DBO), diâmetro biparietal (DBP), comprimento da escápula (CE), comprimento do úmero $(\mathrm{CU})$, comprimento do fêmur (CF), comprimento da tíbia (CT), diâmetro renal transversal (RIMT) e frequência cardíaca (FC). As imagens obtidas durante os exames ultrassonográficos foram salvas no aparelho e posteriormente arquivadas em computador.

A espessura do endométrio foi determinada através da mensuração do diâmetro da parede uterina da fêmea suína gestante, enquanto que o diâmetro da vesícula gestacional foi definido pela medida das extremidades mais distantes obtidas através da visibilização da secção sagital da mesma.

O comprimento crânio-caudal foi encontrado mensurando-se a distância entre a borda rostral do crânio e a borda caudal do acetábulo.

O diâmetro do tórax foi encontrado em secção horizontal, medindo-se a distância entre os bordos ecogênicos das duas últimas costelas. Os diâmetros abdominais transversais e anteroposterior foram avaliados em secção transversal do feto.

O globo ocular foi mensurado através do diâmetro biorbital, do diâmetro orbital e diâmetro interorbital. $\mathrm{O}$ primeiro consiste na medida entre as faces laterais de ambas as órbitas, o segundo compreende a mensuração entre a face lateral e a face medial de um mesmo globo ocular, localizado entre a porção anecogênica do globo ocular e a região hiperecogênica que esta em volta do mesmo e o terceiro corresponde a diastância entre as faces mediais das órbitas.

O diâmetro biparietal foi determinado através da mensuração da maior distância visibilizada entre os ossos parietais que se apresentam hiperecóicos, em secção horizontal do crânio, contrastando internamente com o córtex cerebral que se apresenta hiperecóico, permitindo identificar os dois hemisférios cerebrais e o septo inter-hemisférico.

O comprimento de ossos longos, tais como úmero, rádio, fêmur e tíbia, foi aferido mensurando-se as diáfises. A escápula foi mensurada através do comprimento da espinha da mesma.

O diâmetro renal compreendeu na mensuração transversal das extremidades mais distantes obtidas através da visibilização do mesmo em secção sagital.

A frequência cardíaca foi aferida em modo-M a partir da localização das quatro câmaras cardíacas em modo-B.

\section{RESULTADOS E DISCUSSÃO}

O exame ultrassonográfico em modo-B que, comparado ao eco-ultrassom e ao doppler, é considerado mais adequado e preciso (ALMEIDA, 2002) e, nesse estudo, permitiu realizar diagnóstico gestacional aos 24 dias (Fig. 2), concordando com os achados de CORTEZ et al. (2006) que referem 
visibilização das vesículas embrionárias entre 17 e 27 dias de gestação; no entanto, os mesmos autores referem que, a partir de 21 dias após a cobertura ou IA, a confiabilidade do exame é maior.

Figura 2. Imagem ultrassonografica das vesículas gestacionais evidenciando o embrião (seta) em uma porca com 24 dias de gestação.

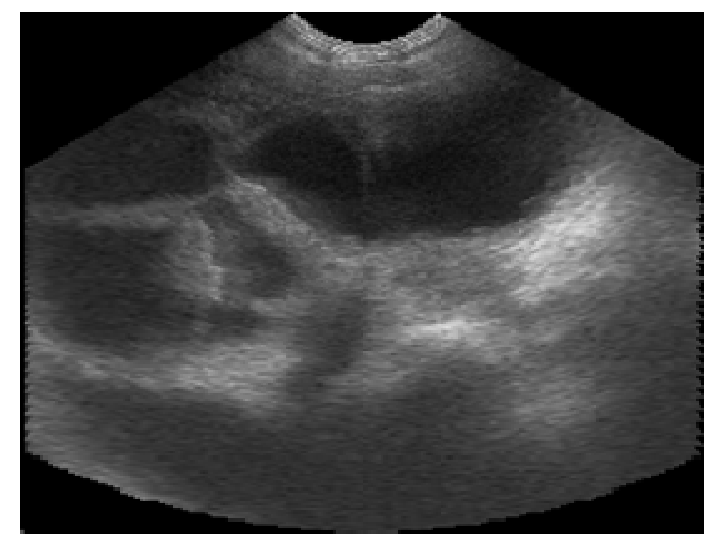

Fonte: Setor de Suinocultura. FAFRAM. Ituverava-SP. 2010.

Comparando-se com outras espécies, em éguas, a gestação pode ser determinada precocemente aos nove dias pós-ovulação usando-se transdutor de boa resolução, mas o período típico para o primeiro diagnóstico geralmente ocorre de 13 a 15 dias pós-ovulação, o que representa uma vantagem de até 4 dias sobre a palpação retal (LEY, 2006). Nas vacas, a gestação pode ser detectada precocemente aos 17 dias após a ovulação usando-se a US em modo-B (BALL; PETERS, 2006). Em ovelhas, a US transretal apresenta-se como método eíicaz, sendo possível diagnosticar a gestação ao $20^{\circ}$ dia após a cobertura, porém com maior acurácia entre os dias 31 e 35 por via transabdominal (CALAMARI et al., 2003; TORRES, 2008; CAVALCANTI et al., 2009). Em caprinos, a calcificação dos ossos fetais aparece com aspecto hiperecóico a partir de 46 dias de gestação, sendo primeiramente identificados costelas e crânio, seguido da coluna vertebral e esqueleto apendicular, período em que é possível realizar a biometria fetal (LÉGA et al., 2003).

Em pequenos ruminantes, a US por via transretal pode diagnosticar mais precocemente a gestação quando comparada à técnica transabdominal (CAVALCANTI et al., 2009), mas em suínos, a via transretal é menos prática por tratar-se de animal confinado e com grande quantidade de fezes, além de apresentarem cornos uterinos muito compridos e posicionados na região mesogástrica, o que dificulta a palpação e a introdução do transdutor através do canal retal (PEQUENO et al., 2009), sendo necessário, para tanto, a utilização de cabo acoplador.

O endométrio foi avaliado a partir da visibilização do conteúdo uterino anecóico proveniente do acúmulo de líquidos fetais e hiperplasia das glândulas endometriais. Sua espessura pode variar dependendo do estado fisiológico, da presença ou ausência de processo inflamatório ou infeccioso e do tamanho do animal (KEALY; McALLISTER, 2005).

A vesícula gestacional é identificada como uma estrutura anecóica vagamente circular, no interior 
da qual está o embrião ecogênico (Fig. 2), característica importante no diagnóstico diferencial entre gestação e afecções uterinas ( PEREIRA et al., 2001; NYLAND; MATTOON, 2005 ). Neste estudo, observou-se que diferentes secções puderam ser realizadas nas vesículas gestacionais, sendo o diâmetro transversal e longitudinal da vesícula embrionária e do saco gestacional, medidas confiáveis para correlacionar a idade gestacional no primeiro terço da gestação (TORRES, 2008). Contudo, para mensuração, considera-se sempre o maior diâmetro da vesícula gestacional (DVG) que deve ser aferido pelo menos três vezes e sua média calculada para que os resultados sejam mais acurados (LÉGA et al., 2007). Assim, a biometria pode ser mais fidedigna na determinação da idade fetal.

Conforme o produto cresce e se calcifica, o esqueleto pode ser identificado como estrutura hiperecóica com sombreamento acústico (KEALY; McALLISTER, 2005). Nesse estudo, foi possível visibilizar estruturas ósseas como o DBP e o CCC a partir de 37 dias de gestação; o DBP foi a variável com maior número de observações e com maior facilidade de visibilização e mensuração, sendo também considerado o parâmetro mais fidedigno na avaliação da biometria fetal em outras espécies (ALMEIDA, 2002, LÉGA et al., 2007, DUPRÉ, 2009).

O CCC pode ser mensurado a partir da identificação do embrião e deve ser aferido no seu maior eixo, porém, a partir da calcificação fetal, este parâmetro deve ser aferido em seção sagital (LÉGA et al., 2003). A identificação dos crânios permite contagem de fetos com maior acurácia do que a avaliação do esqueleto (KEALY; McALLISTER, 2005), no entanto, em fêmeas multíparas, devido às dimensões espaciais da distribuição fetal na cavidade uterina, esse parâmetro pode levar à sobreposição de imagem e dificuldade de aferição (DUPRÉ, 2009). Em pequenos animais, o melhor método para determinação do número de fetos ainda é o exame radiográfico, porém com o inconveniente da possibilidade de promover efeitos mutagênicos (LÉGA et al., 2003; KUSTRITZ, 2005), além de ser pouco viável em animais de produção. Os diâmetros orbiculares incluem diâmetro orbital, diâmetro biorbital e diâmetro interorbital, no entanto, somente o primeiro e o segundo foram aferidos neste estudo.

O diâmetro do tórax (Fig. 3), aferido pela distância entre as últimas costelas com o feto em posição horizontal, pode ser associado a diâmetros abdominais com intuito de estimar o peso fetal (LÉGA et al., 2003), o que pode direcionar futuras pesquisas neste sentido.

Figura 3. Imagem ultrassonográfica em seção horizontal de feto suíno, evidenciando diâmetro torácico (seta).

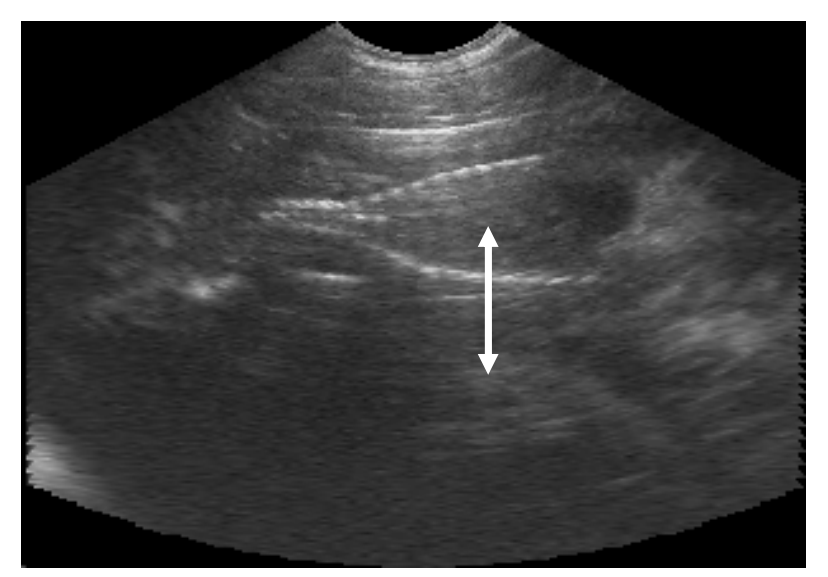

Fonte: Setor de Suinocultura. FAFRAM. Ituverava-SP. 2010.

Nucleus Animalium, v.4, n.2, nov.2012 
A avaliação do comprimento dos ossos longos (CU, CF, CT) deve ser realizada considerando as diáfises ósseas e para o comprimento da escápula (CE), a crista da mesma, que aparecem com o aspecto hiperecóico e também são parâmetros considerados confiáveis na determinação da idade e avaliação da morfologia dos fetos (ALMEIDA, 2007; DUPRÉ, 2009). No presente estudo, os ossos longos (Fig. 4) foram mensurados a partir do $65^{\circ}$ dias de gestação, no entanto, é valido ressaltar que a movimentação fetal ou o tamanho diminuto destes ossos antes deste período, pode dificultar tal aferição (LÉGA et al., 2003).

Figura 4. Imagem ultrassonográfica em seção longitudinal de ossos longos (setas) com aspecto hiperecóico em feto suíno.

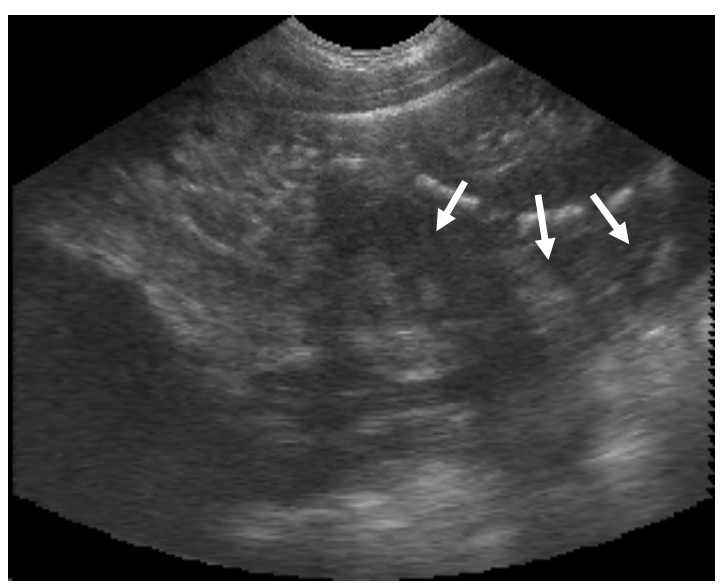

Fonte: Setor de Suinocultura. FAFRAM. Ituverava-SP. 2010.

Os movimentos somáticos e cardíacos são parâmetros variáveis importantes para avaliação da viabilidade e do perfil biofísico fetal (ALMEIDA, 2007; LÉGA et al., 2007). Pela investigação ultrassonográfica é ainda possível avaliar espessura, tamanho e formato do coração (KEALY; McALLISTER, 2005) (Fig. 5); de tônus muscular e movimentos de deglutição que ocorre pela ingestão de líquido amniótico pelo feto, o que confere ao estômago aspecto anecóico (Fig. 1F) e permite desenvolvimento da mucosa gástrica (FARROW, 2006). A frequência cardíaca tende decrescer com o avanço da idade gestacional (MORENO et al., 1996; LÉGA et al., 2007; DUPRÉ, 2009). Neste estudo, houve variação de 205 a 115 batimentos por minutos no período compreendido entre as três últimas semanas da gestação. Frequências muito baixas podem indicar sofrimento fetal (FARROW, 2006; ALMEIDA, 2007). 
Figura 5. Imagem ultrassonográfica em modo $B$ do coração (seta) em seção sagital (A) e imagem ultrassonográfica em modo $\mathrm{M}$ (B) do traçado proveniente da contração do músculo cardíaco em um feto suíno.

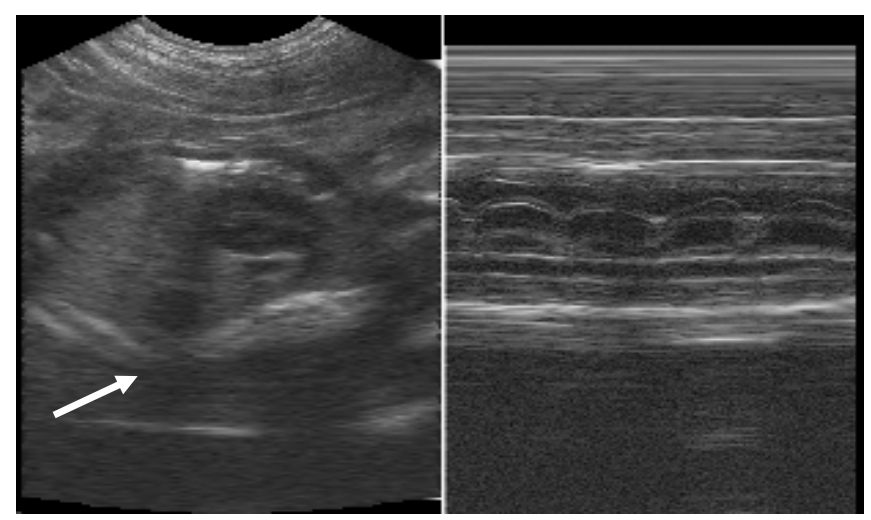

Fonte: Setor de Suinocultura. FAFRAM. Ituverava-SP. 2010.

A visibilização dos rins fetais (Fig. 6) só é possível em estádio gestacional avançado (BONILLAMUSOLES, 1984), o que pode inclusive ser csisiderado como aspecto n portante para se estabelecer início da maturação fetal. Assim, neste estudo, os rins foram visibilizados e mensurados aos 106 dias de gestação, em sua maior seção longitudinal, através da qual é possível diferenciar nitidamente a região medular, do córtex e da pelve renais (ALMEIDA, 2007). A arquitetura renal deve ser sempre considerada como parâmetro morfológico.

Figura 6. Imagem ultrassonográfica em seção sagital de feto suíno evidenciando o rim $(\rightarrow)$ e o estômago $(--\rightarrow)$.

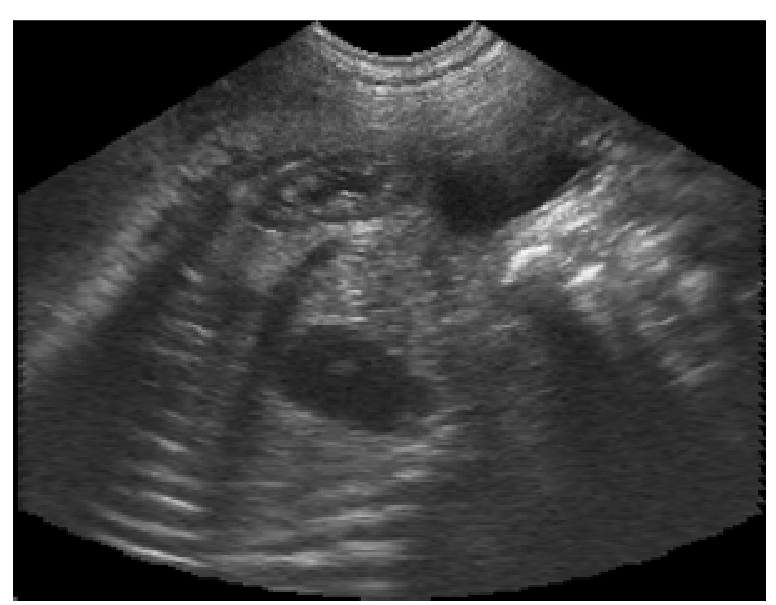

Fonte: Setor de Suinocultura. FAFRAM. Ituverava-SP. 2010.

A fetometria realizada por US demostrou ser uma técnica apropriada para determinação da idade gestacional em suínos, sendo as medidas considerad'is fidedignas para fetos hígidos, neste estudo, visto que as datas de IA e de parto foram conhecidas e que a higidez fetal foi avaliada no momento do nascimento. Porém, por não serem encontrados valores passíveis de comparações em virtude da falta 
de estudos dessa natureza na espécie suína, tais parâmetros podem tornar-se fonte de pesquisas para a espécie.

\section{CONCLUSÃO}

A ultrassonografia em modo-B foi eficaz no diagnóstico gestacional e na avaliação da biometria e morfologia fetais em porcas a partir de 24 dias após a inseminação artificial. O diâmetro biparietal teve maior número de mensurações quando comparado às demais variáveis, demonstrando ser parâmetro fidedigno para determinação da idade fetal. No entanto, novos estudos devem ser realizados em suínos comparando-se a biometria fetal nas diferentes raças e em diferentes estádios gestacionais.

\section{REFERÊNCIAS}

ABREU, D. R. G. Diagnóstico de gestação e fetometria durante os primeiros 90 dias de gestação em caprinos da raça Serrana. 123f. 2006. Relatório Final de Estágio (Trabalho de conclusão de curso de graduação). Universidade de Trás-os-Montes e Alto Douro. Portugal.

ABUD, S. F. A. et al. Uso da ultra-sonografia em fêmeas suínas submetidas a acasalamento natural ou inseminação artificial. Nucleus Animalium, v.1, n.1, p.115-128, 2009.

ALMEIDA, A. H. Estimativa do dia do parto em cadelas da raça Boxer por meio de mensurações ultra-sonográficas no concepto. 2002. 109f. Dissertação (Mestrado). Faculdade de Medicina Veterinária e Zootecnia. Universidade de São Paulo.

ALMEIDA, A. C. Ultra-sonografia. In: NOBREGA, A. I. Tecnologia radiológica e diagnóstico por imagem. 2.ed. São Caetano do Sul: Difusão Editora, 2007. v.4. p.65-97.

ANUALPEC. Anuário da pecuária brasileira. São Paulo: Agra FNP Pesquisas Ltda., 2010. p.253-267.

BALL, P. J. H.; PETERS, A. R. Reprodução em bovinos. 3.ed. São Paulo: Roca, 2006. p.140-142.

BONILLA-MUSOLES, F. Atlas de ultra-sonografia obstétrica. São Paulo: Roca, 1984. 225p.

BRETZLAFF, K. N.; ELMORE, R. G.; NUTI, L. C. Use of na enzyme immunoassay to determine concentrations of progesterone in caprine plasma and milk. Journal of American Veterinary Medicine Association, v.194, n.5, p.664-668, 1993.

CALAMARI, C. V.et al. Avaliação de dois métodos de diagnóstico precoce de gestação em ovelhas: ultrasonografia transretal e detector de prenhez para pequenos ruminantes (DPPR-80®). Brazilian Journal of Veterinary Research and Animal Science, v.40, n.4, p.261-266, 2003.

CAVALCANTI, R. M. et al. Comparação entre dois métodos ultrassonográficos para diagnóstico de gestação em ovelhas da raça Santa Inês. In: CONGRESSO BRASILEIRO DE BUIATRIA, 8, Belo Horizonte-MG, 2009. Ciência Animal Brasileira, supl.1, p.410-416, 2009.

CORTEZ, A. A.et al. Uso do eco-ultrassom, doppler e ultra-sonografia modo-B para o diagnóstico precoce de gestação em suínos. Ciência Veterinária nos Trópicos, v.9, n.1, p.9-16, 2006. 
COSTA, E. T. Equipamentos de auxílio ao diagnóstico por ultrassom. In: CALIL, S. J. Equipamentos médico-hospitalares e o gerenciamento da manutenção: capacitação à distância. Comunicação e Educação em Saúde. Ministério da Saúde, Secretaria de Gestão de Investimentos em Saúde, Projeto REFORSUS. Brasília: Ministério da Saúde, 2002. p.363-398.

DUPRÉ, A. S. A. Avaliação da idade gestacional através da fetometria pelo método de ultrassonografia em ovelhas da raça Hampshire Down (Ovis aries, Linnaeus - 1758). 2009. $55 f$. Dissertação (Mestrado). Faculdade de Medicina Veterinária e Zootecnia. Universidade de São Paulo.

FARROW, C. S. Veterinária: diagnostico por imagem do cão e do gato. São Paulo: Roca, 2006. p.716718.

FELICIANO, M. A. R. et al. Novas perspectivas no diagnóstico ultra-sonográfico gestacional em cadelas: revisão de literatura. Clínica Veterinária, v.13, n.73, p.56-60, 2008.

FERNANDES, T. P. Características ultra-sonográficas em modo-B (tempo real) da gestação na cabra doméstica (Capra hircus LINNAEUS, 1758). 1996. 96f. Dissertação (Mestrado). Faculdade de Medicina Veterinária e Zootecnia. Universidade de São Paulo.

GRANADOS, L. B. C.; DIAS, A. J. B.; SALES, M. P. Aspectos gerais da reprodução de caprinos e ovinos. 2006. Online. Disponível em: <http://www.capritec.com.br/pdf/reproducaode ovinosecaprinos.pdf>. Acesso: 03 out. 2010.

JAINUDEEN, M. R.; HAFEZ, E. S. E. Diagnóstico da gestação. In: HAFEZ, E. S. E.; HAFEZ, B. Reprodução animal. 7.ed. Barueri-SP: Manole, 2004. p.399-408.

KAUFFOLD, J.; RICHTER, A.; SOBIRAJ, A. Results and experiences of a two-year investigations in the use of sonographic pregnancy control in sows at different stages of gestation. Tierarztlliche Praxis. Ausgabe G, Grosstiere/Nutztiere, v.25, n.5, p.429-435, 1997.

KEALY, J. K.; McALLISTER, H. Radiologia e ultra-sonografia do cão e do gato. 3.ed. Barueri-SP: Manole, 2005. 436p.

KUSTRITS, M. V. R. Pregnancy diagnosis and abnormalities of pregnancy in the dog. Theriogenology, v.64, n.3, p.755-765, 2005.

LÉGA, E. Acompanhamento ultra-sonográfico transabdominal em modo-B e concentração sérica de progesterona dos primeiros 60 dias de gestação na cabra doméstica (Capra hircus, Linnaeus, 1758). 2000. 95f. Dissertação (Mestrado). Faculdade de Ciências Agrárias e Veterinárias. Universidade Estadual Paulista. Jaboticabal.

LÉGA, E; TONIOLLO, G. H.; RESENDE, K. T. Acompanhamento ultra-sonográfico transabdominal em modo-B dos primeiros 60 dias de gestação na cabra doméstica. Ars Veterinaria, v.19, n.2, p.156-165, 2003.

LÉGA, E. et al. Determinação da idade fetal por meio da técnica ultra-sonográfica de fetometria e de morfologia fetal em cabras. Arquivo Brasileiro de Medicina Veterinária e Zootecnia, v.59, n.4, p.851856, 2007.

LEY, W. B. Reprodução em éguas: para veterinários de equinos. São Paulo: Roca, 2006. p.124-126.

MACHADO, M. M. et al. História da ultra-sonografia intra-operatória. Radiologia Brasileira, v.35, n.6, p.351-355, 2002.

Nucleus Animalium, v.4, n.2, nov.2012 
MORENO, J. C.; CHAVEZ, C. S.; BICKHARDT, K. Fetale herzfrequenzmessung und sonografhische fetometrie zur bestimmung des trächtigkeitsstadiums bei schaf. Deutsche Tierarztliche Wochenschrift, v.103, n.11, p.478-480, 1996.

NYLAND, T. G.; MATTOON, J. S. Ultra-som diagnóstico em pequenos animais. 2.ed. São Paulo: Roca, 2005. 506p.

PEQUENO, A. P.; ZÚNIGA, C. E. A.; WISCHRAL, A. Utilização do ultrassom modo-B no estudo do sistema reprodutivo de fêmeas suínas. Revista Brasileira de Reprodução Animal, v.33, n.3, p.161-168, 2009.

PEREIRA C. Z.et al. Sincronização da ovulação em fêmeas suínas submetidas ao desmame precoce. Arquivo Brasileiro de Medicina Veterinária e Zootecnia, v.53, n.4, 2001.

PETER, A. T.; JAKOVLJEVIC, S. Real-time ultrasonography of the small animal reproductive organs. Compendium on Continuing Education for the Practicing Veterinarian, v.14, n.6, p.739-746, 1992.

REICHLE, J. K.; HABEL, G. K. Ultrasonic biparietal diameter of second trimester Pygmy goat fetuses. Theriogenology, v.35, n.4, p.689-694, 1991.

ROMERO, C. A. Utilización práctica del ecógrafo de pantalla como método de diagnóstico de gestación en ganado porcino. 2006. Online. Disponível em:

<http://albeitar.portalveterinaria.com/noticia.asp?ref=3552\&pos=616>. Acesso: 03 out. 2006.

SILVEIRA, P. R. S. et al. Manejo da fêmea reprodutora. In: SOBESTIANSKY, J.; WENTZ, I.;

SILVEIRA, P. R. S.; SESTI, L. A. C. Suinocultura intensiva: produção, manejo e saúde do rebanho. Brasília: Embrapa-SPI; Concórdia: EMBRAPA-CNPSA, 1998. p.177-181.

TORRES, C. A. Estimação da idade gestacional por ultra-sonografia no primeiro terço da gestação em cabras Saanen. 2008. 54f. Dissertação (Mestrado). Faculdade de Veterinária. Universidade Estadual do Ceará. Fortaleza.

VIANA, C. H. C. et al. Diagnóstico de cistos ovarianos pela palpação de ultra-sonografia transretal em fêmeas suínas: relato de dois casos clínicos. Brazilian Journal of Veterinary Research and Animal Sciense, v.35, n.2, p.64-68, 1998.

WENZEL, J. G. W. Exame clínico do sistema reprodutivo: suínos. In: RADOSTITS, O. M.; MAYHEW, I. G. J.; HOUSTON, D. M. Exame clínico e diagnóstico em veterinária. Rio de Janeiro: Guanabara Koogan, 2002. p.566.

WILLIAMS, S., PINEIRO, P., DE LA SOTA, R. L. Ultrasonografía reproductiva en producción porcina. Analecta Veterinaria, v.21, n.1, p.50-56, 2001.

WOLF, A.; GABALDI, A. S. Acompanhamento ultra-sonográfico da gestação em grandes animais: parte II. Ciências Agrárias e da Saúde, v.2, n.2, p.84-89, 2002. 
